\title{
TRACHEOSTOMY MANAGEMENT DURING THE COVID -19 PANDEMIC
}

\section{BHESANIYA ND ${ }^{1 *}$, NANIWADEKAR RG ${ }^{2}$ AND PATIL DA}

1: Junior Resident, Department of Surgery, Krishna Institute of Medical Sciences Deemed To Be University, Karad, Maharashtra (India

2: Professor, Department Of Surgery, Krishna Institute of Medical Sciences Deemed To Be University, Karad, Maharashtra (India)

3: Senior Resident, Department of Surgery, Krishna Institute of Medical Sciences, Deemed to be University, Karad, Maharashtra (India)

*Corresponding Author: Dr. Nikunj D Bhesaniya: E Mail: Dr.n.d.bhesaniya@gmail.com

Received $12^{\text {th }}$ July 2021; Revised $14^{\text {th }}$ Aug. 2021; Accepted $27^{\text {th }}$ Oct. 2021; Available online $15^{\text {th }}$ Feb. 2022

\section{https://doi.org/10.31032/IJBPAS/2022/11.2.1020}

\begin{abstract}
The coronavirus 2019 pandemic has lead to never existing demands on the modern health care system, and the highly transmissible nature of the virus has led to particular concerns of infection among health care workers. While strong data regarding the contagious nature of the infection aren't yet widely available, preliminary information suggests risk of transmission among asymptomatic individuals, including those within health care facilities. Among patients with unsuspected infection tracheostomy presents a risk of droplet infection. In this study, Multidisciplinary open airway working group has developed guideline for open airways at our hospital providing practical \& technical guidance.
\end{abstract}

\section{INTRODUCTION}

The COVID-19 pandemic has resulted in world to satisfy this overwhelming unparalled numbers of patients admitted demand [1]. Mechanical ventilation is to medical care units (ICUs) with vital for roughly $68 \%$ of patients supplementary capacity created in admitted to ICU with medial LOS various hospitals everywhere in the (Length of Stay) of 14 days (IQR 7-20 
days) [2]. Tracheostomy is

usually performed by critical care specialities to enable ventilator wean when mechanical ventilation is continous (7-10 days) [3, 4]. Result of this pandemic has lead to need for tracheostomy in significant number of patient $[5,6]$.

Advantage of tracheostomy include the ability to knock effect of sedation, moderately decreasing ventilatory support, improve communication, take part in rehabilitation, lessens dead space, and certainly deminish complications like vocal fold granuloma formation, subglottic stenosis $[\mathbf{7 ,} 8]$. However, there are recognised complications and risks to the procedure which can be harder to manage in the pandemic situation.

COVID-19 unfolds via direct contact and droplet routes hence deliberate measures should be taken while doing tracheostomy procedure is of prime significance to avert cross-contamination between patients and hospital staff. Transference risk is expanded by blending of viral particles with air [9, 10]. Manoeuvres done for Airway administration by healthcare workers (HCW) are identified as aerosol generating procedures (AGPs), and these may present a significant risk of COVID-
19 transmission [10]. Procedures generating aerosol during tracheostomy care $[9,10,11,12,13,14]$.

Manifestation, timing, capability and delivery of tracheostomy care such direction is dominant to contribute the straight forward standard of care, extremely as health care personnels redeployed into ICU to administer the surge in patient volumes may lack apposite expertness.

We strike back to this instant need of caring tracheotomised patients being treated for COVID-19 pneumonitis, with prominence in the tracheostomy care and the process of weaning and decannulation. Providing proper practical guideline for Management of tracheotomised patient in COVID-19 infection is paramount aim of this paper.

Procedures generating aerosol during tracheostomy care

1. Open suction of the respiratory tract

2. Tracheostomy-related decannulation and care procedures Induction of Sputum

3. Fiberoptic examination of upper respiratory tract Bronchoscopy

4. Changing of Tracheostomy tubes

5. Progress between ventilator-free and supportive mechanical ventilation during weaning 
6. Heat and moisture exchange filters changing.

\section{Infection risk}

Acknowledgement of the hazard of suffering to $\mathrm{HCW}$ has developed since the COVID-19 pandemic began in Wuhan at the top of 2019. A comprehensive survey of COVID-19 infection rates in $\mathrm{HCW}$ will surely come after within the near future, but recent reports from Italy suggest that HCW are disproportionately affected (healthcare worker account for $8.4 \%$ cases [15]) and identical rates reported recently by Choou et al. [16]. The coronavirus isn't considered to be an airborne virus so airborne precautions aren't routinely required [17]. However, certain procedures associated with airway create aerosols which remain in the air and increasing risk of transmission to distances beyond 2 metres.

The risk of COVID-19 infection with AGPs is that hospitals around the planet have been clear in their guidance that precautions must be followed to protect healthcare personnel. This adds the use of PPE like disposable gloves, fluidrepellent gown, filtering face piece respirator and eye or face protection. AGPs should be carried out in one room with negative pressure airflow whenever feasible and shut the doors with minimum health care worker present. Full personal protective equipment would want to be worn to enter the space for a period of your time after the AGP counting on the air change rate; within the absence of a negative pressure room, this room isolation period would wish extending

A good hospital ventilation supplies certain viral particle clearance. One air exchange removes around $65 \%$ of the virus [18]. After 5 air exchanges in the room, there is less than $1 \%$ of the original viral load. Five exchanges take around $25 \mathrm{~min}$ in most clinical surroundings. Nonetheless, in ICU or in operation room, the air exchange is much quick and it takes only $12 \mathrm{~min}$ for five exchanges. While negative pressure rooms are endorsed for AGPs (aerosol generating procedures), our experience has shown that availability of such settings are difficult to get. In some segments of hospital, recodification can alter a positive pressure room or whole ward to negative pressure. Room which has fresh air and good ventilation is very dominant than whether it is positive or negative pressure [19].

The sensitivity of swab testing is not $100 \%$ yet. Hence, patient with a negative swab result does not exclude the need for full PPE [17]. Moreover, 
patients with a tracheostomy could also be infectious for extended period of time than the special COVID-19 patient.

This is why tracheostomy patients will, by definition, be critically ill during their episode of COVID-19 and this is correlated with detained cleared viral RNA [19]. The expanded sensitivity of COVID-19 noticing in blind endotracheal aspirates compared to salivary and orophaingeal swab tests suggests that the viral burden is elevated within the lower tract. Tracheostomy exposes HCW through exposure to these endobronchial sections during routine care. These factors, integrated with the putative peak viral load in early disease, weigh the agreement for later tracheostomy in COVID-19 patients.

\section{Basic tracheostomy care}

The concept of care applies to a tracheostomized patient who is suspected or diagnosed with a case of COVID-19 infection as well as to any other tracheostomized patient in the hospital. Best tracheostomy care means providing best care which include following emergency protocols for trancheostomy; tracheostomy care; humidification; Inflation of cuff; oral suctioning and oral hygiene.

During recovery phase begins process of rehabilitation which includes good communication between patient and HCW, Early weaning and decannulation [20, 21]. To provide effective care cooperation of all specialities is critical. This is where benefits of a multidisciplinary tracheostomy team come to play who work together in tandem with standardised guidelines.

\section{Emergency situations}

Early recognition of emergency situations and rapid management are critical for all tracheostomy care, this becomes more important in the patient with COVID-19 positive status. Regular monitoring of procedures should happen at regular interval to prevent imminent emergencies. Cohorted care of "positive" patients may help to overcome risk from delays in putting on and taking off PPE. Otherwise, the location of patients in side rooms with doors closed may regulate communication and may delay ingress to equipment. Therefore, it is required to count this time while aoting staff to tracheostomized patients.

Unless all staff during procedure are wearing PPE, no airway-related procedures are per- formed. This include all staff entering the ICU/ward should be in full PPE. In situation where HCWs not wearing appropriate PPE, modified mechanisms of emergency care need to be started. For the moment remaining 
team makes ready to attend the emergency in full PPE kit. Supplemental equipment may have to be provided as standard and other mechanisms for increasing team communication should be considered.

\section{Open Airway Management}

We define open airways as a deacon of laryngectomy or bronchiectomy, paying careful attention to those not associated with a closed ventilation circuit. In these cases, we adopted methods to create a closed system as described by Chan et al. [9]. While not necessarily eliminating the possibility of an aerosol, relatively simple measures may significantly reduce droplet spread and protect the supply of the healthcare workforce.

For patients with tracheostomy, partial closure may be most easily achieved with a tracheostomy cap in those who can tolerate them, although this may be uncommon in an incompatible setting. An attached tracheostomy heat exchanger (HME) can also provide an easy solution as a droplet barrier. Various HME devices are commercially available but may not be locally available in individual institutions or may also be out of stock due to supply shortages

Various improvised systems can be designed to address these issues with the stocks available in most institutions. HME from the anesthesia cycle is often adapted to tracheostomy if HME tracheostomy is not available (image no.1). A closed inline suction system are often attached to the tracheostomy tube before the HME, and such a system will allow a safer means of suctioning without frequent opening of the airway. This would also prevent the HME from becoming dipped as quickly as without an in-line suction system (at $1 \mathrm{~B}$ and $1 \mathrm{C})$.

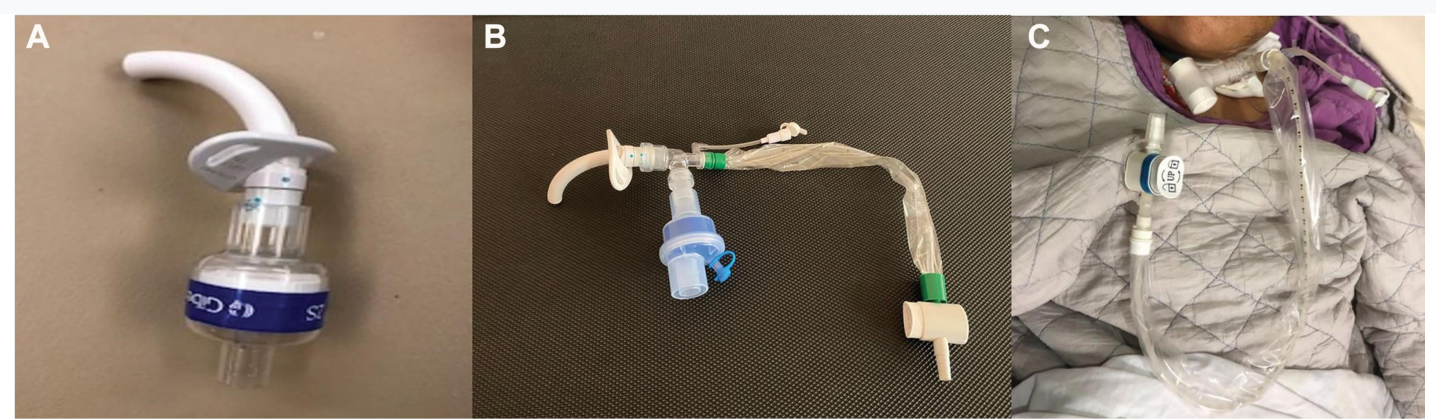

Image 1: Methods of closing the tracheostomy circuit. (A) A heat moisture exchanger are often connected on to a tracheostomy. $(B, C)$ An in-line suction are often added for safer suctioning. 


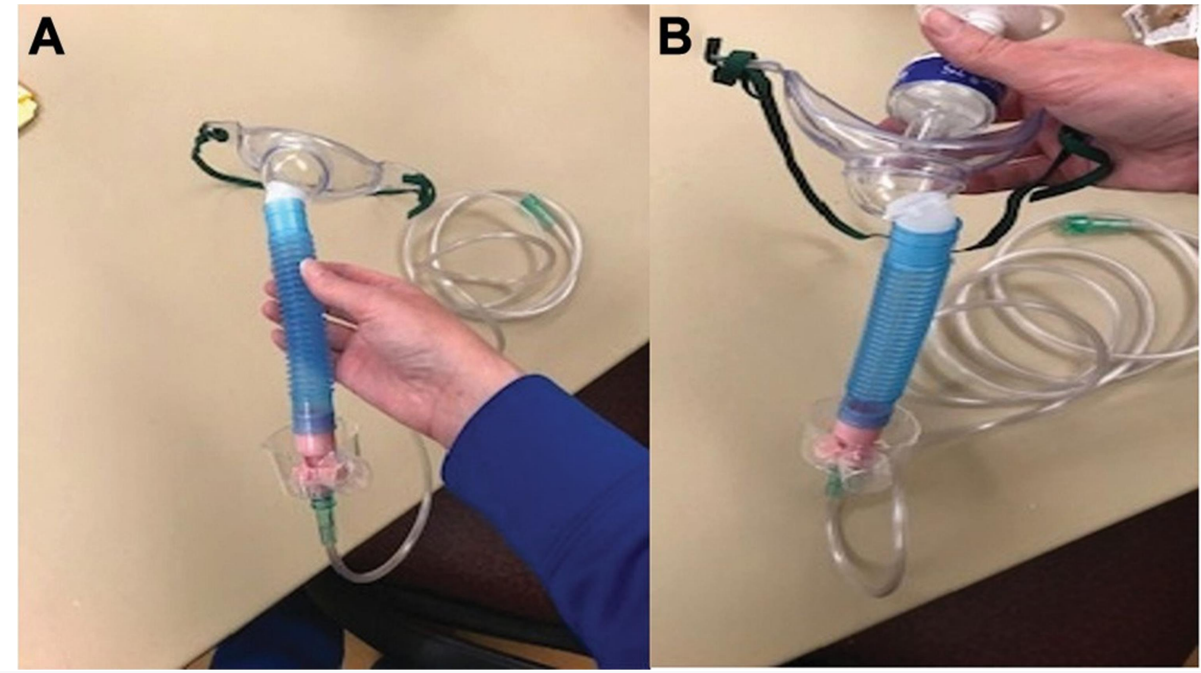

Image 2: If oxygen therapy is required, it can be connected to (A) a tracheostomy mask, which can then be connected to (B) a heat exchanger (HME)

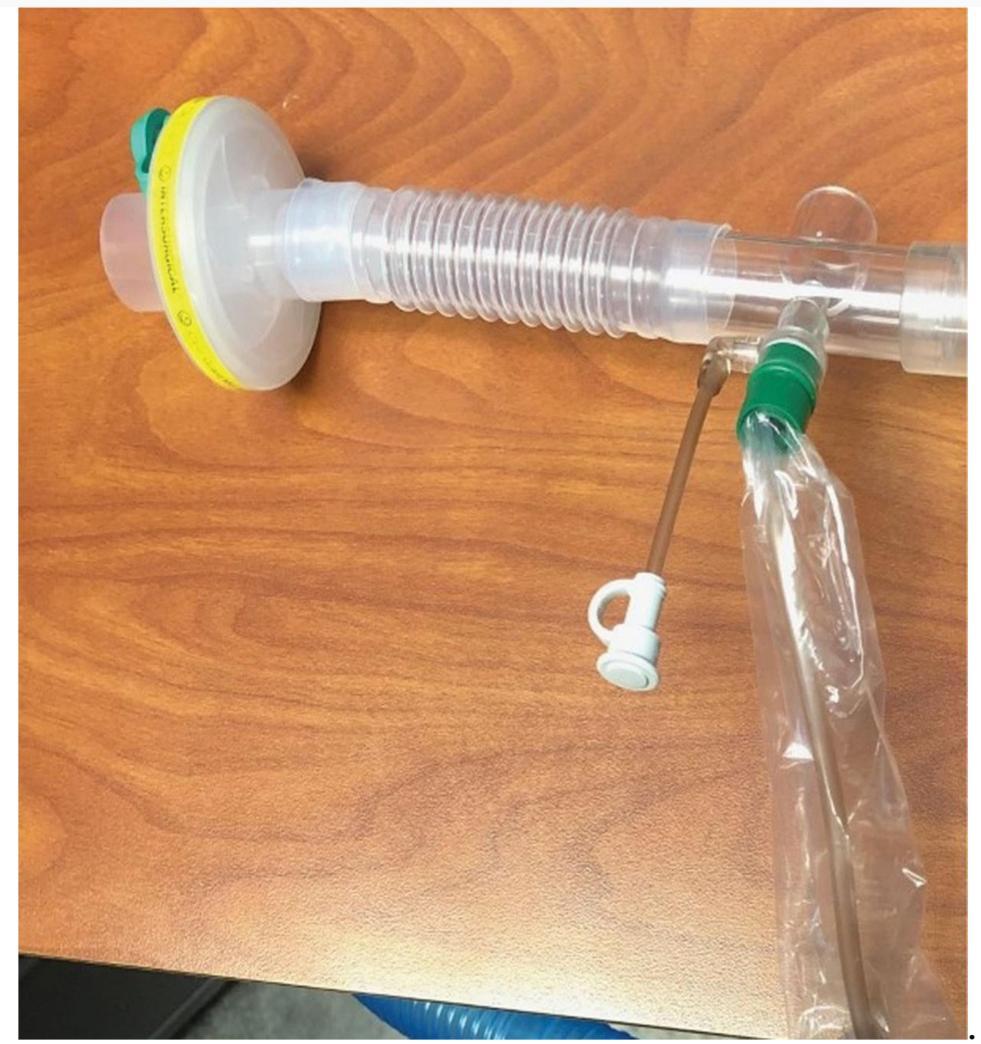

Image 3: Oxygen therapy and in-line suction can be combined in this tracheostomy setting.

In patients with laryngectomy, an adhesive base plate or silicone laryngectomy tube with an attached HME cassette provides a similar benefit and is well tolerated. Handcuffs may also be used on the tracheostomy tube within the stoma, provided that tracheostomy adaptations are also used.

For open airways within an outpatient setting or if these systems are not tolerated, 
a surgical airway mask or tracheostomy mask / collar will provide some protection. When possible, multi-channel zero cannula tubing should be avoided. Internal cannulas require regular brushing, which poses a danger of aerosolizing respiratory particles. The use of disposable inner cannulas is preferred since they will be discarded and replaced. Caution is taken in the deployment of these systems because some patients may become intolerant or the system may become disrupted. If patients are unable to demonstrate the ability to safely get rid of the system, then additional monitoring may also be appropriate, such as continuous pulse oximetry and telemetry. They can create extra torque on the tracheostomy tube, which could increase the risk of accidental disconnection, pressure ulcers, or perhaps decannulation.

Commercially available connections designed to stabilize the fan circuit or further tick around the neck and closure system can help reduce this risk. Additional personal protective equipment (PPE) and isolation precautions can be guaranteed in the management of open airways in a respiratory pandemic, but specific recommendations need to be established at the institutional level and are beyond the scope of this communication.
Advanced planning for managing tracheotomised COVID-19 patients

Successful and quick daring requires an experienced multidisciplinary staff; Therefore, it could be ideal to cohort tracheostomized patients to facilitate methodical intake. However, it is unclear whether patients discharged with ICU still return positive strikes for COVID-19 viral RNA and remain contagious [22]. However, site-specific measures depending on ward ergonomics and ventilation are needed to effectually segregate COVID-19 positive and negative patients.

In our hospital so far, the capacity and status of the patient has been such that most patients have progressed to decannulation while still in the cohort "COVID-19 positive" level 3 of it (data to be published).

Tracheostomy management simulation training

$\mathrm{HCW}$ is being diverted in large numbers to medical units and designated COVID19 wards. For many, this has guide to the requirement to put up to the care of patients with unknown requirement including tracheostomy management. The simulation consequently has a vital role to train hospital staff both in the procedure and in the subsequent management of the tracheostomies [23]. 
Examples of procedural simulations adds corpse-based training to show surgeons the percutaneous tracheostomy technique. Examples of department-based simulations include trial of clinically based scenarios with high-fidelity dummies. Trainings can use a variety of teaching methods to develop skills and overcomes risk. Conceiving a program that incorporates a composition of didactic learning and hand-to-hand simulation has proven demanded. Sessions necessitate all relevant members of the multi-disciplinary team to confirm the scenarios are as realistic as possible.

Advanced patient simulators are frequently used with scenarios assisted real obstacles and played call at real time. Decision-making skills and team work as well as communication skills can also be practised. Although practically it can be harder to create clinical scenarios in a non-clinical environment.

\section{Weaning and decannulation process}

Weaning from ventilation support is important goals of tracheostomy. Tracheostomy venture will restore physiological respiration, with its benefits of filtering, warming and moistening the inhaled air. Daring is achieved with progressive reductions in support pressure.
Prior to COVID-19, regular periods of deflation of cuffs were used to see for gag reflex, motivate for swallowing into the mouth. However, from these steps there is the potential of both droplets and aerosol generation when on the ventilator, so the decannulation process should be changed to move through these phases only when the patient can tolerate self-ventilation. Although lowering the tracheostomy tubes is not a routine practice, it may be necessary to enable withdrawal for patients who are not progressing.

However, proper risk management of this may be required as tube tracheostomy removal and AGP insertion are necessary. This concatenation of events leads to the final step; tracheostomy decannulation. Every organization should have its own decanlation protocol that reflects local expertise, experience and resources.

During these unparalled times, every effort must be made to speed up the decannulation of patient sales after ventilator support is no longer required. This enables effectual patient care and lowers the pressure on our stretched healthcare system. A tracheostomy team consisting of nurses, speech and language therapists, respiratory physiotherapists, critical care specialists, respiratory 
physicians, and head and neck surgeons is crucial to achieve this, especially when led by overworked staff.

The health care acknowledge to the COVID-19 pandemic will most likely cause an higher number of tracheotomized patients in our hospitals. Many contentions still exists in the management of patients with COVID-19 infection as researchers having difficulty keeping pace with the quickly progressing disease.

Randomized consequence studies comparing patients with contrasting care strategies or use of equipment, while providing strong evidence, necessary time and assests that are not sensible within a COVID 19 pandemic environment. Consequently, we present a practical proposal to tracheotomized patient care, weaning and decannulation. This is supported by the good quality evidence accesible and interpreted in light of experience of COVID-19 patients. It is evident that it is of the greatest significance to make sure the safety of patients and staff and it requires one across the board team for all the patients undergoing tracheostomy throughout recovery from COVID-19. Finally, we suggest continuous possible data collection and monitoring to deface future practices.

\section{CONCLUSION}

The rapid spread of COVID-19 has presented massive challenges to healthcare facilities, and our adjustments must be just as rapid despite inadequate information and resources. These open airway management guidelines are elements of a prudent strategy to keep the health workforce supported by development information. Surgeons have been at the forefront of identifying these risks and will need to be leaders in the skirmish of responses within their institutions

\section{Acknowledgement}

I am thankful to Mrs Rupali Salunkhe for secretariat help, Institute of Medical Sciences Deemed To Be University, Karad, Maharashtra (India)

\section{REFERENCES}

[1] Phua J, Weng L, Ling L, Egi M, Lim CM, Divatia JV, et al., Intensive care management of coronavirus disease 2019 (COVID19): challenges and recommendations, The lancet respiratory medicine, 8(5), 2020, 506-170, Intensive Care National Audit and Research Centre (2020).

[2] ICNARC report of COVID-19 in critical care, 10, 2020, 2020.

[3] Griffiths J, Barber VS, Morgan L, Young JD, Systematic review and 
meta-analysis of studies of the timing of tracheostomy in adult patients undergoing artificial ventilation, Bmj, 330(7502), 2005 , 1245.

[4] Adly A, Youssef TA, El-Begermy MM, Younis HM, Timing of tracheostomy in patients with prolonged endotracheal intubation: a systematic review, European Archives of Oto-RhinoLaryngology, 278(3), 2018, 679-90.

[5] Angel L, Kon ZN, Chang SH, Rafeq S, Shekar SP, Mitzman B, et al. $\quad$ Novel percutaneous tracheostomy for critically ill patients with COVID-19, The Annals of thoracic surgery, 110(3), 2020, 1006-11.13

[6] Takhar A, Walker A, Tricklebank S, Wyncoll D, Hart N, Jacob T, et al., Recommendation of a practical guideline for safe tracheostomy during the COVID-19 pandemic, European of Oto-RhinoLaryngology, 277(8), 2020, 217488.

[7] Nagendra $S$, Trehan S, Tracheostomy: A shift in paradigm from a simple bedside procedure to a potential source of spread of COVID-19, Education in the Health Professions, 56(2), 2021, 79.
[8] Shinn JR, Kimura KS, Campbell BR, Lowery AS, Wootten CT, Garrett CG, et al., Incidence and outcomes of acute laryngeal injury after prolonged mechanical ventilation, Critical care medicine, 47(14), 2019, 1698.

[9] Chan JY, Wong EW, Practical aspects of otolaryngologic clinical services during the 2019 novel coronavirus epidemic: an experience in Hong Kong, JAMA otolaryngology-head \& neck surgery, 147(7), 2020, 520-21.

[10] Tran K, Severn M, Conly J, AGPs and risk of transmission of acute respiratory infections to healthcare staffs: a systematic review, PloS one, 7(4), 2013, e35897.

[11] Thamboo A, Lea J, Sowerby L, Abdalkhani A, Diamond C, Ham $\mathrm{J}$, et al., Clinical evidence based review and recommendations of aerosol generating medical procedures in otolaryngologyhead and neck surgery during the COVID-19 pandemic, Journal of Otolaryngology-Head \& Neck Surgery 49(1), 2021, 1-5.

[12] Chung FF, Liu HE, Lien AS, Hsiao HF, Chou LT, Aerosol distribution during open suctioning and long-term 
surveillance of air quality in a respiratory care center within a medical center, Respiratory care, 60(1), 2016, 30-7.

[13] Thompson KA, Pappachan JV, Mittal H, Macken S, Nguyen-VanTam JS, Copley VR, et al., Influenza aerosols in UK hospitals during the H1N1 (2009) pandemic-the risk of aerosol generation during medical procedures, PloS one, 8(3), 2001, e56278.

[14] Lazzerini M, Putoto G, COVID-19 in Italy: momentous decisions and many uncertainties, The Lancet Global Health, 8(5), 2021, e642-2.

[15] Chou R, Dana T, Buckley DI, Selph S, Fu R, Totten AM, Epidemiology of and risk factors for coronavirus infection in health care workers, Annals of internal medicine, 173(3), 2020, 120-36.

[16] Wang J, Liu F, Zhou M, Lee YF, Will the status of infection prevention and control (IPC) professionals be improved in the context of COVID-19?

[17] Coia JE, Ritchie L, Adisesh A, Booth CM, Bradley C, Bunyan D, et al., Guidance on the use of respiratory and facial protection equipment, Journal of hospital Infection, 85(4), 2014, 171-85.

[18] Cook TM, El-Boghdadly K, McNarry AF, Patel A, Higgs A, Consensus guidelines for managing the airway in patients with COVID-19: the Faculty of Intensive Care Medicine and the Royal College of Anaesthetists, Anaesthesia, 75(6), 2020, 785-99.

[19] Dawson D, Essential principles: tracheostomy care in the adult patient, Nursing in critical care, 19(2), 2014, 46-72.

[20] Kutsukutsa J, Kuupiel D, MonoriKiss A, del Rey-Puech P, Mashamba-Thompson TP, Tracheostomy decannulation methods and procedures for assessing readiness for decannulation in adults: a systematic scoping review, JBI Evidence Implementation, 17(2), 2019, 74-92.

[21] Bolton L, Brady G, Coffey M, Haines J, Roe J, Wallace S, RCSLT guidance: Speech and language therapist-led endoscopic procedures in the COVID-19 pandemic, R Coll Speech Language Ther Expert Panel, 2020, 2-7. 
[22] Freeman-Sanderson AL, Togher L, Elkins M, Kenny B, Quality of life improves for tracheostomy patients with return of voice: a mixed methods evaluation of the patient experience across the care continuum, Intensive and Critical Care Nursing, 46, 2018, 10-7. 